\title{
Long noncoding RNA MAFG-AS1 facilitates bladder cancer tumorigenesis via regulation of $\mathrm{miR}-143-3 p / S E R P I N E 1$ axis
}

\author{
Xi Sun, Yi Cai, Xiheng Hu, Miao Mo, Cheng Zhao, Wei He, Yangle Li \\ Department of Urology, Xiangya Hospital, Central South University, Changsha, China \\ Contributions: (I) Conception and design: Y Li; (II) Administrative support: M Mo; (III) Provision of study materials or patients: X Sun, Y Cai; (IV) \\ Collection and assembly of data: X Hu, C Zhao; (V) Data analysis and interpretation: W He; (VI) Manuscript writing: All authors; (VII) Final \\ approval of manuscript: All authors approved the final manuscript. \\ Correspondence to: Yangle Li. Department of Urology, Xiangya Hospital, Central South University, No. 87 Xiangya Road, Changsha 410008, China. \\ Email: liyangle2003@163.com.
}

\begin{abstract}
Background: Numerous studies have shown that long noncoding RNAs (lncRNAs) act as key regulators of bladder cancer progression. While lncRNA MAFG-AS1 has been confirmed as an oncogenic molecule in various cancers and tumorigenesis, in present study, we investigated its function and role in the tumorigenesis of bladder cancer.

Methods: The expression of MAFG-AS1, miR-143-3p and SERPINE1 in bladder cancer tissues was detected by qRT-PCR methods. The relationship between MAFG-AS1 expression and clinicopathologic characteristics of bladder cancer was analyzed. The effects of MAFG-AS1 depletion on cell proliferation, migration, invasion and apoptosis were investigated. The binding relationship of MAFG-AS1, miR-143-3p and SERPINE1 was examined by luciferase reporter analysis and RNA immunoprecipitation (RIP) assay.

Results: MAFG-AS1 was upregulated in bladder cancer tissues and cell lines. High MAFG-AS1 expression was associated with bladder cancer histological grade, TNM stage and lymph node metastasis, and patients with high expression showed poor overall survival. Cell function experiments showed that MAFG-AS1 silencing markedly suppressed bladder cancer cell proliferation, migration, invasion and increased cell apoptosis. Moreover, our results demonstrated that MAFG-AS1 functioned as a competing endogenous RNA for miR-143-3p to modulate SERPINE1 levels. Further analysis showed that miR-143-3p inhibition or SERPINE1 overexpression alleviated the suppressive effects of MAFG-AS1 silencing on malignant features.

Conclusions: Our findings indicated that MAFG-AS1 facilitates tumorigenesis via regulation of the miR-143-3p/SERPINE1 axis and also provides a novel insight into tumorigenesis and identify a promising therapeutic target for bladder cancer.
\end{abstract}

Keywords: Bladder cancer; tumorigenesis; MAFG-AS1; miR-143-3p; SERPINE1

Submitted May 01, 2020. Accepted for publication Sep 20, 2020.

doi: $10.21037 /$ tcr-20-1971

View this article at: http://dx.doi.org/10.21037/tcr-20-1971

\section{Introduction}

Bladder cancer is the ninth most common carcinoma worldwide, with more than 430,000 patients diagnosed with each year (1). Transitional cell carcinoma is the main histological type, accounting for over $90 \%$ of bladder cancer cases. For early and mid-stage bladder cancer patients, surgery is the optimal treatment is suitable, frustratingly, it is prone to relapse. For patient with metastases, palliative treatments such as radiotherapy and chemotherapy are the most common treatment strategy (2). Although the diagnosis and treatment of bladder cancer have been made significant advances, the 5 -year survival for invasive patients are only $50 \%$, furthermore, the recurrence rate of bladder cancer is $15-90 \%$ within 5 years $(2,3)$. Therefore, understanding the molecular mechanisms of bladder cancer 
progression will help to identify possible targets for cancer therapy.

Long noncoding RNAs (lncRNAs) are transcripts that are $>200$ nucleotides long (4). Although they do not code proteins, they can regulate gene expression at the transcriptional and post-transcriptional levels and epigenetic modifications (5). An increasing number of studies have demonstrated that lncRNAs participate in the tumorigenesis and progression of numerous cancers, which been the focus of anti-tumour therapy (6-8). For example, IncRNA LNMAT1 contributes to lymphatic metastasis by epigenetic activation of CCL2 in bladder cancer (9). Chen et al. (10) reported that IncRNA OXCT1-AS1 acts as an miR-455-5p sponge to inhibit its binding to JAK1, leading to elevated JAK1 expression and enhanced bladder cancer aggressiveness. Dai et al. (11) demonstrated that lncMUC20-9 impeded bladder cancer cell growth, migration and invasion via suppression of ROCK1. However, the functions and underlying mechanisms of lncRNAs in bladder cancer progression remain unclear.

Aberrant expression of the novel lncRNA MAFG antisense RNA 1 (MAFG-AS1) was recently identified in a variety of cancers and facilitated cancer progression (12-16). However, its role and underlying mechanisms remain unclear in bladder cancer. Basing on the TCGA database analysis, MAFG-AS1 was found upregulation in bladder cancer tissues, more importantly, patients with high expression of MAFG-AS1 showed poor overall survival. It implied that MAFG-AS1 play an importance role in bladder cancer.

In present research, we demonstrated that MAFGAS1 promotes bladder cancer cell proliferation, migration and invasion via competitive inhibition of miR-143-3p and elevation of SERPIN1 levels. Our findings provide a novel insight into tumorigenesis and reveal a promising therapeutic target for bladder cancer.

We present the following article in accordance with the MDAR reporting checklist (available at http://dx.doi. org/10.21037/tcr-20-1971).

\section{Methods}

\section{Clinic samples}

A total of 52 pairs of bladder cancer tissue samples and corresponding adjacent normal tissues were obtained from Xiangya Hospital, Central South University from Oct 2018 to Oct 2019. All patients enrolled in this research didn't receive any preoperative therapy. This study was conducted in accordance with the Declaration of Helsinki (as revised in 2013) and approved by the institutional ethics committee of Xiangya Hospital, Central South University (approval No. 2019032542). Informed consent has been signed by all patients before specimen collection. The pathological features were acquired from patients' medical records. Specimen were fast frozen in liquid nitrogen and kept at $-80{ }^{\circ} \mathrm{C}$.

\section{Cell culture and transfection}

Human bladder cancer cell lines (J82, 5637, UM-UC-3 and T24) and normal cell SV-HUC-1 were obtained from COBIOER (Nanjing, China). J82 and UM-UC-3 were maintained in Minimum Eagle's medium containing 10\% FBS. 5637 and T24 were maintained in RPMI1640 supplemented with $10 \%$ FBS. SV-HUC-1 were cultured in F-12K medium containing $10 \%$ FBS.

SiRNAs targeting the lncRNA MAFG-AS1 sequences were purchased from GenePharma (Shanghai, China). The sequences information as shown in Table 1. Scramble oligonucleotides were used as a negative control. MiR-1433 p mimics and inhibitor were obtained from GeneCopoeia (Guangzhou, China). SERPINE1 cDNA ORF plasmid was purchased from Sino Biological (Beijing, China). Cells were transfected by Lipofectamine 2000 (Thermo Fisher, USA) following the manufacturers' instructions. Forty-eight h later, cells were collected for subsequent experiments.

\section{qRT-PCR assay}

RNA isolation was performed using TRIzol reagent (Thermo Fisher, USA). cDNA was synthesized using the GoScript ${ }^{\mathrm{TM}}$ Kit (Promega, USA) following the product's instructions. qRT-PCR analysis was conducted on LightCycler480 system (Roche, Germany). GAPDH and U6 used as internal control for normalizing. Each reaction was conducted in triplicate. The primer sequences were listed in Table 1.

\section{MTT and colony formation assay}

For cell proliferation analysis, approximately $5 \times 10^{3}$ cells were grown in 96-well plates and maintained for $24 \mathrm{~h}$. Then, each well was added $20 \mu \mathrm{L}$ MTT solution and maintained at $37^{\circ} \mathrm{C}$ for $4 \mathrm{~h}$. After removing the supernatant, 
Table 1 The sequences of siRNAs and qRT-PCR primers

\begin{tabular}{ll}
\hline Name & \multicolumn{1}{c}{ Sequence } \\
\hline siRNAs & \\
MAFG-AS1 siRNA-1 & GGGCAAUUCCAACCAAGAAAC \\
MAFG-AS1 siRNA-2 & GCUGCAGUGAGCUGUGAUCAU \\
Scramble siRNA & UUCUCCGAACGUGUCACGU \\
qRT-PCR & \\
MAFG-AS1 forward & GGGACGGAGACAAATGACGG \\
MAFG-AS1 reverse & GCAGGCTCCCTGACACGTA \\
SERPINE1 forward & GAGGATGAAAGAAACAGCCAGCT \\
SERPINE1 reverse & CCCGCTATGAAATAGATTCACGT \\
GAPDH forward & ACACCCACTCCTCCACCTT \\
GAPDH reverse & TTACTCCTTGGAGGCCATGT \\
\hline
\end{tabular}

each well was replenished by $150 \mu \mathrm{L}$ DMSO and maintained at $37^{\circ} \mathrm{C}$ for $10 \mathrm{~min}$. Finally, the absorbance was examined at $570 \mathrm{~nm}$ with a microplate reader (Molecular Devices, USA).

For colony formation experiment, $1 \times 10^{3}$ cells were seeded into a $35 \mathrm{~mm}$ dish and maintained for two weeks. Next, cells were fixed with $4 \%$ paraformaldehyde (PFA) and stained by $0.01 \%$ crystal violet dye.

\section{Wound healing and invasion assay}

For wound healing experiment, cells were cultured in a 12well plate for $24 \mathrm{~h}$ to reach nearly $90 \%$ confluence. Cell wounds were scratched using a sterile pipette tip. Then, cells were washed by PBS 3 times and cultured with serumfree medium. The wound was photographed at 0 and $24 \mathrm{~h}$.

For invasion assay, $1 \times 10^{5}$ cells suspended in serum-free medium were added into the upper chamber coated with Matrigel (Corning, USA), while medium containing 20\% FBS was added into the lower chambers. After maintaining at $37^{\circ} \mathrm{C}$ for $24 \mathrm{~h}$, the non-invading cells were cleaned by cotton swabs, and the invasive cells were fixed by $4 \%$ PFA and stained in $0.1 \%$ crystal violet for $15 \mathrm{~min}$. Finally, the invasive cells were captured under the microscope (Nikon, Japan).

\section{Cell apoptosis assay}

Cells were collected and centrifuged at 2,000 rpm for $5 \mathrm{~min}$. Then cells were incubated with Annexin V-FITC and PI
(BioLegend, USA) according to manufacture protocol. After staining at room temperature in the dark for $20 \mathrm{~min}$, the suspending cells was added with $500 \mu \mathrm{L}$ Binding buffer. Finally, cells apoptosis was analysed by flow cytometry (CytoFlex, Beckman, USA) within 1 hour.

\section{In vivo animal experiment}

Four-to-five-week-old BALB/c nude mice were purchase from Hunan SJA Laboratory Animals Center of the Chinese Academy of Sciences (Changsha, China) and fed according to the guidelines authorized by the Animal Care Committee of the Xiangya Hospital, Central South University. One $\times 10^{6}$ cells suspended in $0.2 \mathrm{~mL}$ PBS were subcutaneously injected into the right flank of animals. Tumor growth was measured and recorded every 5 days. Twenty-five days later, animals were sacrificed after anesthetized and stripped the tumors. Tumor volume was calculated by the formula: $\left(\right.$ length $\times$ width $\left.^{2}\right) / 2$.

\section{Subcellular fractionation}

The nuclear and cytosolic fractions of 5637 and T24 cells were isolated with PARIS Kit (Thermo Fisher, USA) following to the manufacturer's protocol.

\section{Luciferase reporter analysis}

The fragment of MAFG-AS1 and SERPINE1 comprising miR-143-3p binding sites were obtained by PCR and cloned into a psiCHECK-2 vector to construct wildtype psiCKECK2-MAFG-AS1 (Wt-MAFG-AS1) and psiCKECK2-SERPINE1 (Wt-SERPINE1) or mutant psiCKECK2-MAFG-AS1 (Mut-MAFG-AS1) and psiCKECK2-SERPINE1 (Mut-SERPINE1) vectors. 5637 and T24 cells were respectively transfected with Wt or Mut recombinant plasmids, together with miR-NC or miR-143$3 p$ mimics. Forty-eight hours later, the luciferase activity was determined with the Dual-Luciferase reporter assay Kit (Promega, Madison, USA) following the manufacturer's instructions.

\section{RNA immunoprecipitation (RIP) assay}

RIP assay was performed using the EZ-Magna RIP kit (Millipore, USA) according to the protocol. Simply, cells were lysed in RIP lysis buffer containing proteinase and 
RNase inhibitor. The cell lysate was centrifuged after $10 \mathrm{~min}$ of incubation. Then, the cell supernatant extract was incubated magnetic beads pre-conjugated with AGO2 antibody (Millipore, USA) or IgG at $4{ }^{\circ} \mathrm{C}$ overnight. RNA was purified from immunoprecipitation complex and qRTPCR detection was used to evaluate the level of MAFGAS1, miR-143-3p and SERPINE1.

\section{Statistical analysis}

All experiments were repeated three times, and the data were presented by mean \pm standard deviation (SD). The statistical analysis was conducted by SPSS 22.0 (SPSS Inc., IL, USA). Student's $t$-test was applied to analyse the differences between two groups. Chi-square test was performed to evaluate the relationship between MAFGAS1 expression and clinicopathological features of bladder cancer. $\mathrm{P}<0.05$ was considered statistically significant.

\section{Results}

LncRNA MAFG-AS1 was overexpressed in bladder cancer tissues and implied the poor survival

Firstly, qRT-PCR was used to examined MAFG-AS1 expression in 52 paired primary bladder cancer specimen and matched adjacent normal tissues. The result displayed that MAFG-AS1 was obviously upregulated in bladder cancer samples (Figure 1A). Moreover, MAFGAS1 expression in lymph node (LN)-metastatic tissues was obviously higher than nonmetastatic tumor tissues (Figure $1 B$ ). The cutoff value was set basing on the median value of MAFG-AS1 expression in bladder cancer, the patients were divided into the low expression group $(\mathrm{n}=25)$ and the high expression group $(\mathrm{n}=27)$ (Figure 1C). The correlation of MAFG-AS1 expression and pathological characteristics were evaluated. The result displayed showed that the high expression of MAFG-AS1 was obviously associated with histological grade $(\mathrm{P}=0.026)$, TNM stage $(\mathrm{P}=0.012)$, and $\mathrm{LN}$ metastasis $(\mathrm{P}=0.047)$ (Table 2). Besides, MAFG-AS1 overexpressed in bladder cancer tissues was confirmed by GEPIA database (http://gepia.cancer-pku.cn) (Figure 1D). Additionally, high MEST expression indicated the poor overall survival of patients with bladder cancer from Kaplan-Meier plotter (http://kmplot.com/analysis/) (Figure 1E). These data demonstrated that MAFG-AS1 was upregulated in bladder cancer and implied the poor survival.

\section{LncRNA MAFG-AS1 facilitated bladder cancer cell proliferation, migration and invasion}

To determine the role of MAFG-AS1 in bladder cancer, firstly, MAFG-AS1 expression in bladder cancer cells (J82, 5637, T24 and UM-UC-3) and normal uroepithelial cell SV-HUC-1 was analyzed. We observed that MAFG-AS1 were markedly upregulated in tumor cell lines compared with SV-HUC-1 (Figure 2A). Next, MAFG-AS1 silencing in 5637 and T24 cells were performed via transfecting MAFG-AS1 siRNAs. The result demonstrated that MAFGAS1 was obviously downregulated in 5637 and T24 cells (Figure 2B). MTT and clone formation assays showed that MAFG-AS1 silencing significantly inhibited cell proliferation and growth compare with the control group (Figure 2C,D). Wound scratch and invasion assays demonstrated that MAFG-AS1 silencing significantly impeded cell migration and invasion (Figure 2E,F). Flow cytometer detection showed that MAFG-AS1 depletion markedly increased cell apoptosis (Figure 2G). Moreover, MAFG-AS1 depletion significantly suppressed the tumor growth in vivo (Figure $2 \mathrm{H}$ ). These findings suggested that MAFG-AS1 act as an oncogene in bladder cancer progression.

\section{MiR-143-3p targeted MAFG-AS1}

In order to uncover the mechanism of MAFG-AS1 on bladder cancer tumorigenesis, we firstly investigated its subcellular location. We observed that MAFG-AS1 was preferentially enriched in the cytoplasm (Figure $3 A$ ). This result implied that MAFG-AS1 could competitively interact with cytoplasmic miRNAs. So, the potential miRNA targets of MAFG-AS1 were predicted using starBase v3.0. MiR-143-3p was a predicted target and was chosen for verification as it has been reported to be involved in oncogenesis and cancer progression (17-19). The wild type and mutant type of MAFG-AS1 containing miR-143-3p binding site were constructed as showing in Figure 3B. The luciferase activity assay was conducted in 5367 and T24 cells. The result displayed that co-transfection of miR143-3p mimics and Wt-MAFG-AS1 markedly reduced the luciferase activity (Figure 3C). Additionally, RIP experiment was performed to examine the interaction between miR143-3p and MAFG-AS1. MAFG-AS1 and miR-143-3p were significantly enriched in the AGO2 immunoprecipitate comparing with the IgG control group (Figure $3 D$ ).

Next, the expression of miR-143-3p level in the 52 
A

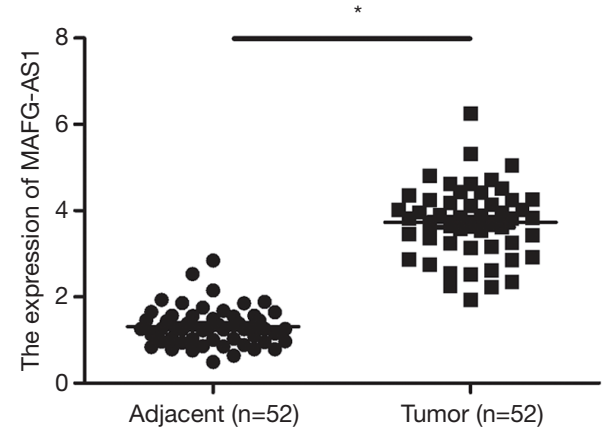

B

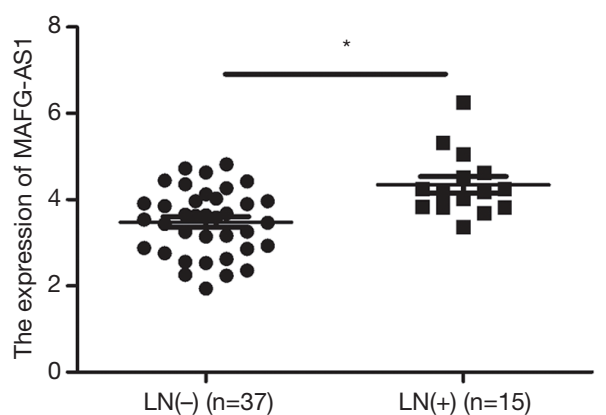

C

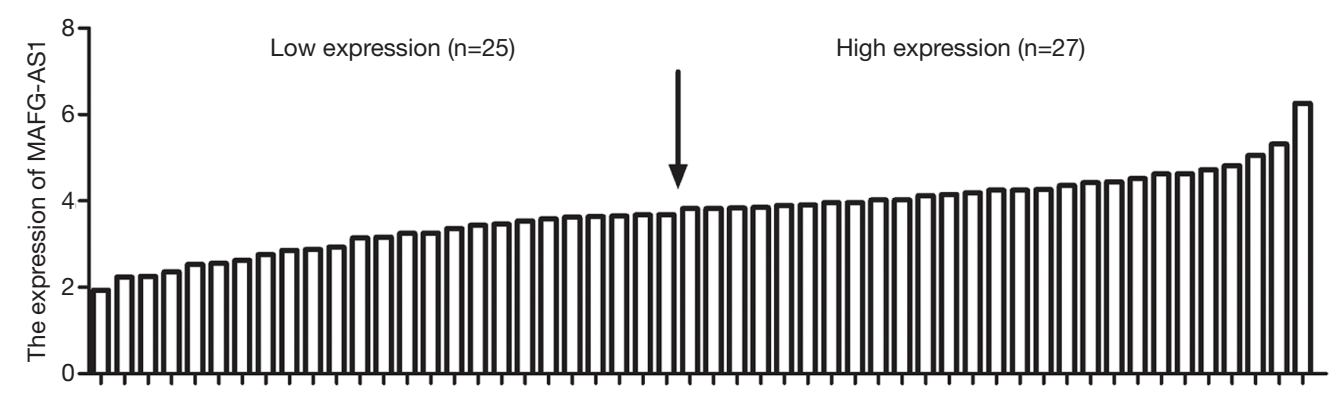

D

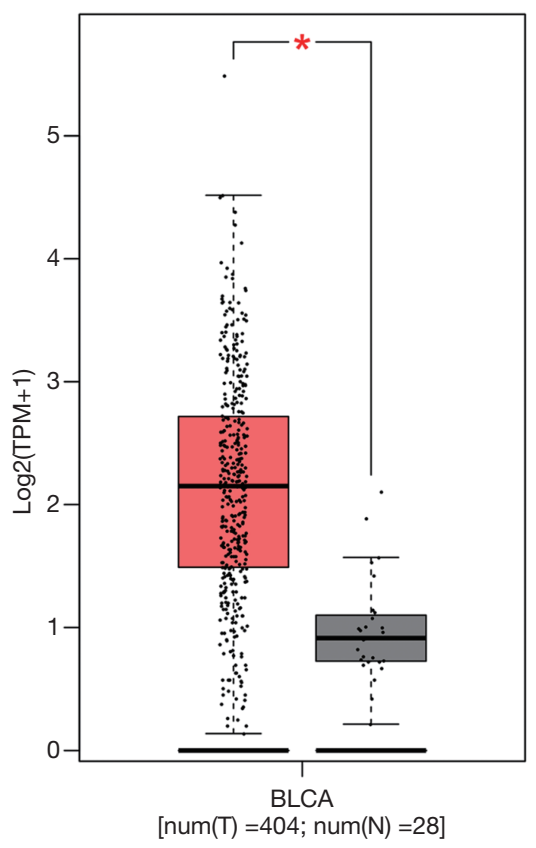

$\mathrm{E}$

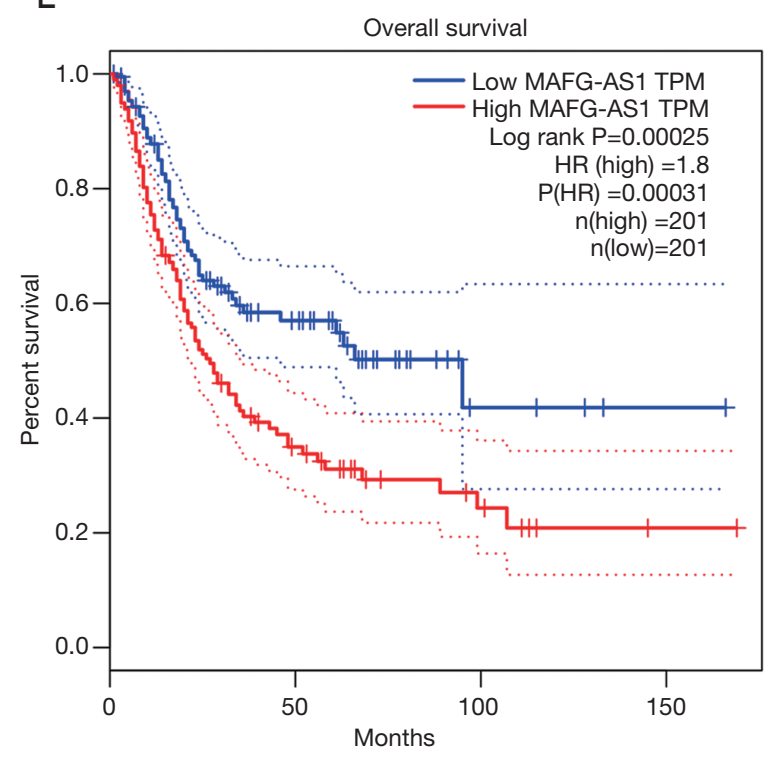

Figure 1 LncRNA MAFG-AS1 was overexpressed in bladder cancer tissues and its high expression indicated the poor survival. (A) qRTPCR examined the expression of MAFG-AS1 in 52 paired primary bladder cancer specimen and matched adjacent normal tissues. (B) The expression of MAFG-AS1 was analyzed in LN metastatic [LN(+)] and nonmetastatic bladder cancer tissues [LN(-)]. (C) Patients with bladder cancer were divided into high-expression and low-expression groups according to the median value of MAFG-AS1 expression. (D) MAFG-AS1 overexpressed in bladder cancer tissues was confirmed by GEPIA database (http://gepia.cancer-pku.cn). (E) The high MEST expression indicated the poor overall survival of patients with bladder cancer from Kaplan-Meier plotter (http://kmplot.com/analysis/). *, $\mathrm{P}<0.05$. 
Table 2 Association between MAFG-AS1 expression and clinicopathologic characteristics in bladder cancer

\begin{tabular}{|c|c|c|c|c|}
\hline Features & Number of cases & \multicolumn{2}{|c|}{ MAFG-AS1 expression } & $P$ value \\
\hline Age (years) & & & & 0.500 \\
\hline$>60$ & 26 & 14 & 12 & \\
\hline$\leq 60$ & 26 & 13 & 13 & \\
\hline Male & 33 & 17 & 16 & \\
\hline Female & 19 & 10 & 9 & \\
\hline Tumor size & & & & 0.508 \\
\hline$>3 \mathrm{~cm}$ & 28 & 15 & 13 & \\
\hline Low & 25 & 9 & 16 & \\
\hline High & 27 & 18 & 9 & \\
\hline TNM grade & & & & $0.012^{*}$ \\
\hline I-II & 28 & 10 & 18 & \\
\hline III-IV & 24 & 17 & 7 & \\
\hline Lymph nodes metastasis & & & & $0.047^{*}$ \\
\hline No & 37 & 16 & 21 & \\
\hline Yes & 15 & 11 & 4 & \\
\hline
\end{tabular}

pairs of bladder cancer samples and matched adjacent normal tissues was analyzed. MiR-143-3p was found downregulation and negatively correlated with MAFG-AS1 expression in bladder cancer samples (Figure 3E,F). Besides, we observed that MAFG-AS1 silencing could evidently elevate miR-143-3p level in 5367 and T24 cells (Figure 3G). Collectively, our result confirmed that miR-143-3p was a target of MAFG-AS1.

\section{SERPINE1 was a target of MiR-143-3p}

Three online softs TargetScan, miRDB and microRNA were performed to identify the possible target genes of miR-143-3p. We found that serpin family member 1 gene (SERPINE1) (also named PAI1) was a candidate target gene (Figure $4 A$ ). In order to confirm it, luciferase reporter analysis was executed. The result displayed that miR-143-3p mimics depressed the luciferase activity of Wt-SERPINE1 but not in the Mut-SERPINE1 (Figure 4B). Moreover, the expression of SERPIN1 was markedly reduced in 5367 and T24 cells transfected with miR-143-3p mimics (Figure 4C). Notably, RIP assay found that miR-143-3p and SERPINE1 were prominently enriched in the AGO2 immunoprecipitate (Figure 3D). Additionally, in bladder cancer tissues, qRT-PCR displayed that SERPINE1 was obviously upregulated and negatively correlated with that of miR-143-3p (Figure 4D,E). Collectively, our results indicated that SERPINE1 was a target of miR-143-3p.

\section{MAFG-AS1 facilitated bladder cells tumorigenesis by regulating miR-143-3p/SERPINE1 axis}

Whether miR-143-3p or SERPIN1 participated in promotive effect on bladder cancer malignant phenotypes mediated by MAFG-AS1, rescue assays were executed. As shown by qRT-PCR, miR-143-3p inhibition evidently reduce the MAFG-AS1 depletion-induced suppressing effect on the SERPIN1 expression. Meanwhile, SERPINE1 

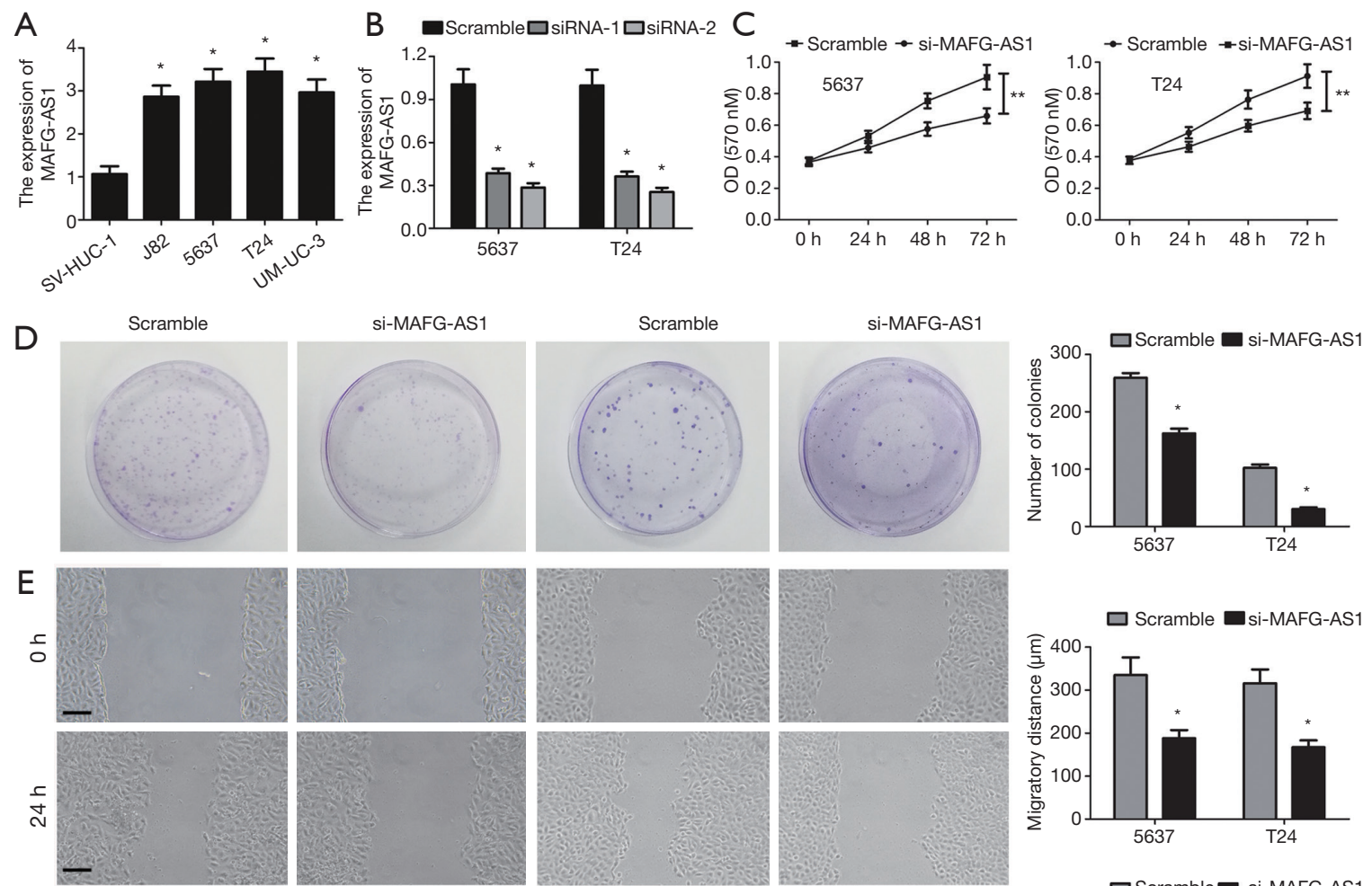

$\mathrm{F}$
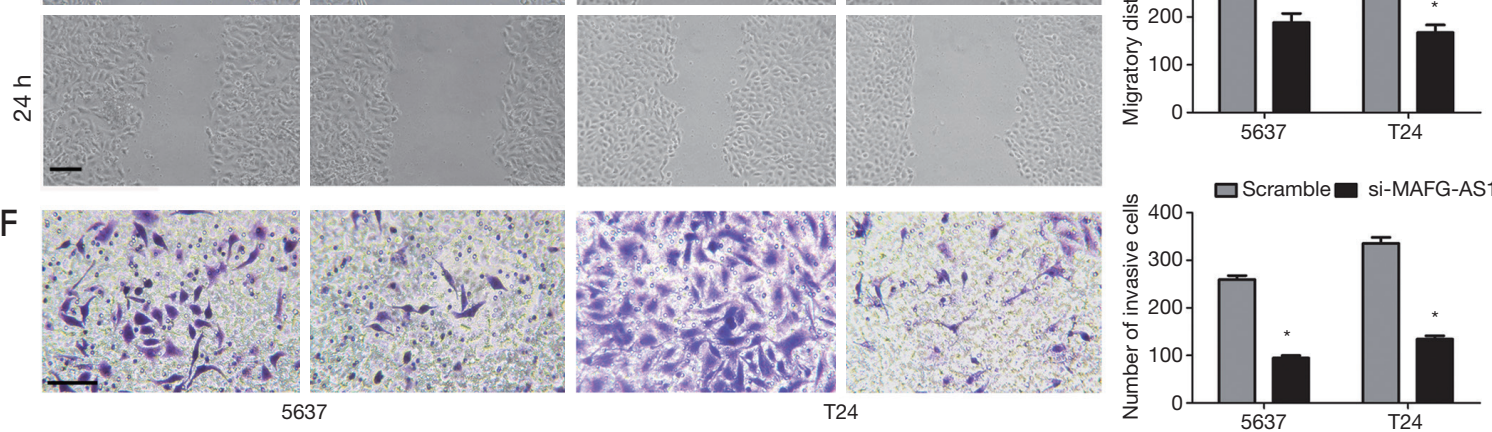

\section{G}
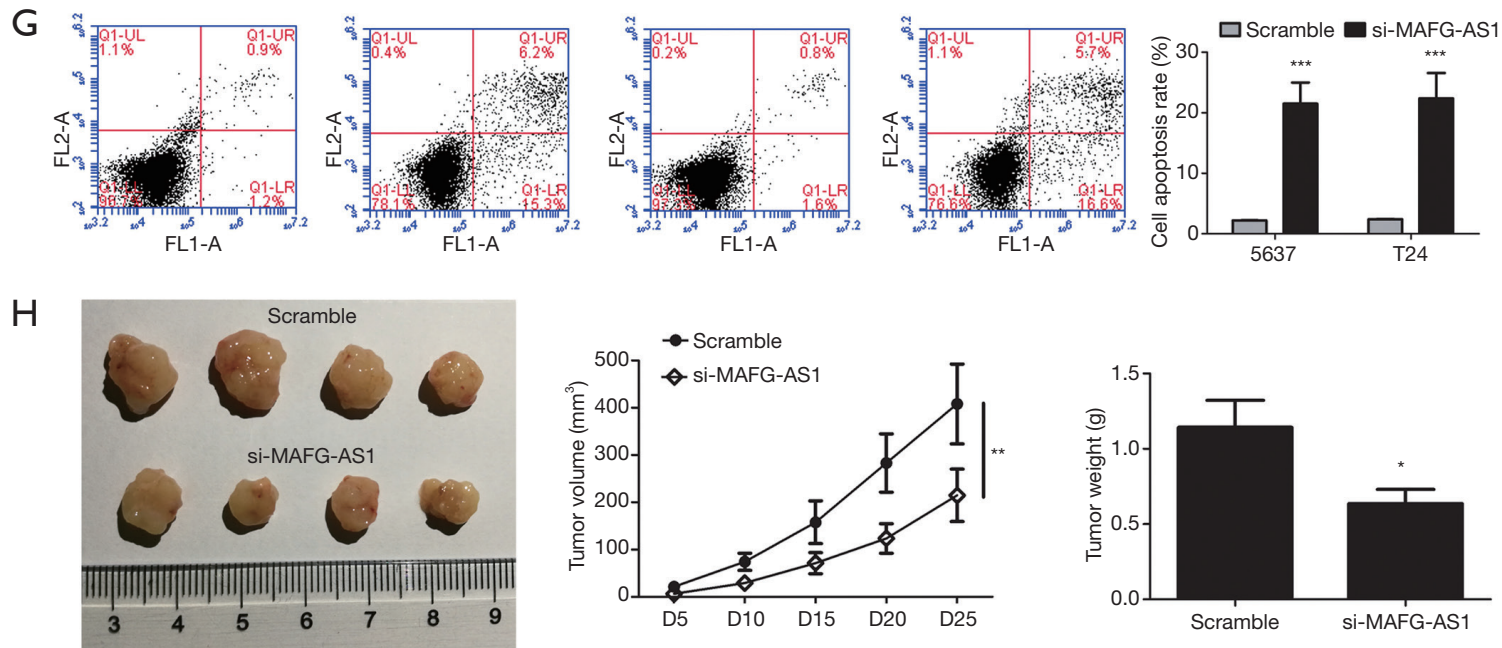

Figure 2 MAFG-AS1 facilitated bladder cancer cell proliferation, migration and invasion. (A) qRT-PCR detected the expression of MAFGAS1 in tumor cell lines (5637, UM-UC-3, T24) and bladder epithelial immortalized cell SV-HUC-1. (B) Expression of MAFG-AS1 were examined in MAFG-AS1 silencing cells. MTT (C), clone formation (D), wound scratch (magnification 100x) (E), Transwell assays (magnification 200×) (F) and cell apoptosis (G) were performed to evaluated the effects of MAFG-AS1 silencing on bladder cancer cell proliferation, migration, invasion and cell apoptosis. $(\mathrm{H})$ MAFG-AS1 knockdown inhibited the tumor volume and weight in nude mice tumorigenicity assay. Scale bars $=100 \mu \mathrm{m},{ }^{*}, \mathrm{P}<0.05 ;{ }^{* *}, \mathrm{P}<0.01,{ }^{* *}, \mathrm{P}<0.001$. 
A

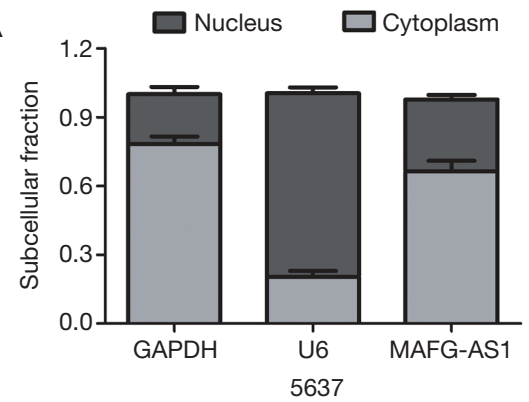

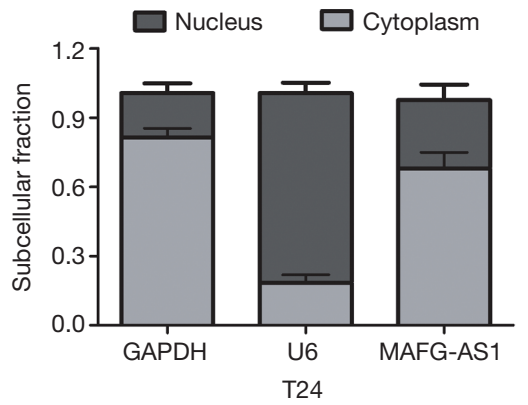

B

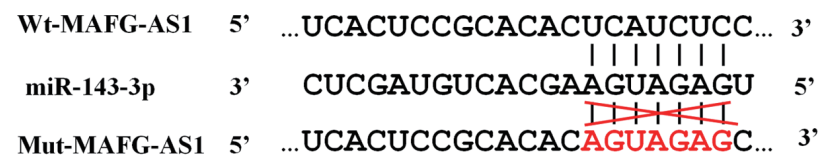

C

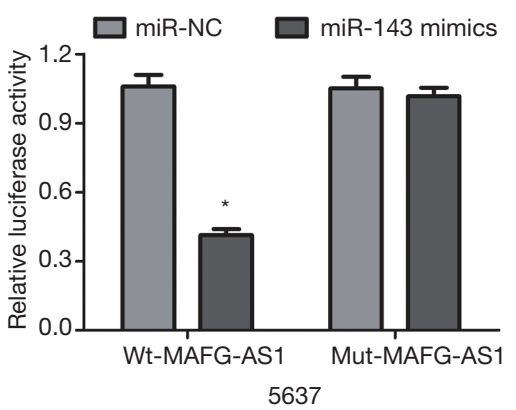

$\mathrm{E}$

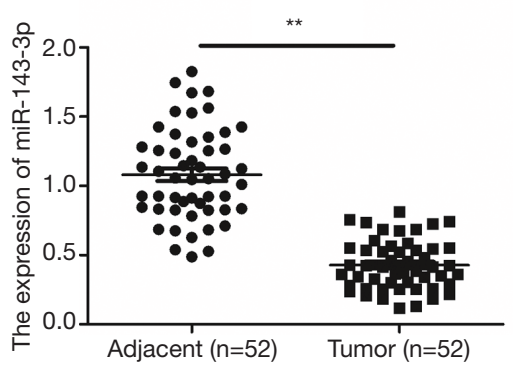

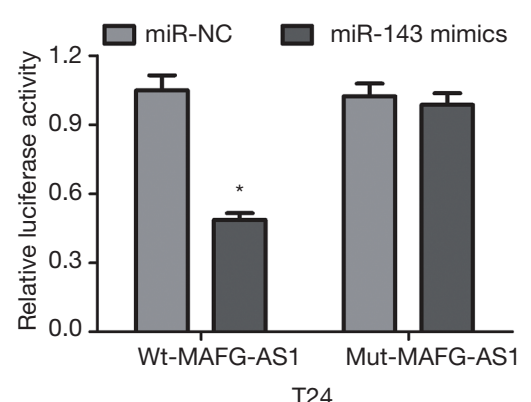

$\mathrm{F}$

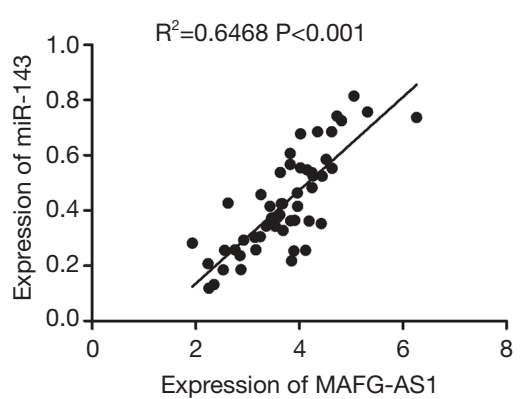

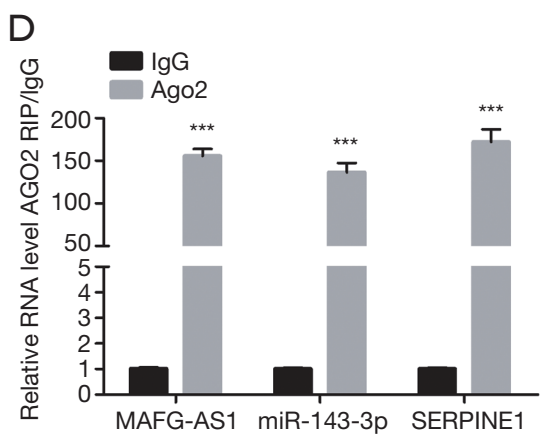

G

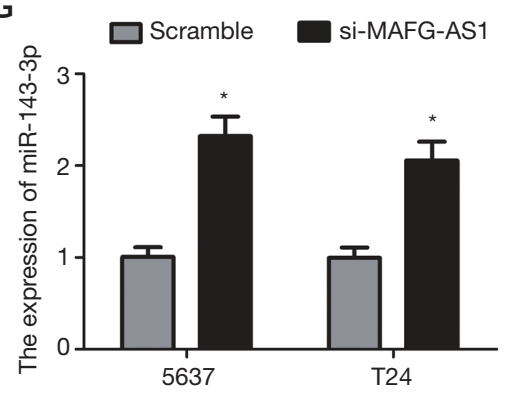

Figure 3 MiR-143-3p targeted MAFG-AS1. (A) Subcellular fractionation analysis displayed that MAFG-AS1was mainly enriched in the cytoplasm. (B) Prediction with starBase 3.0 uncovered the possible binding site for miR-43-3p in MAFG-AS1. (C) Luciferase reporter assay analyzed the luciferase activity of the Wt or Mut MAFG-AS1 reporter in 5637 and T24 cells after transfection of miR-143-3p. (D) RIP assay was performed to examine the interaction between MAFG-AS1, miR-143-3p and SERPINE1. (E) qRT-PCR detected miR-143-3p level in the 52 pairs of bladder cancer samples and matched adjacent normal tissues. (F) Correlation analysis between MAFG-AS1 and miR143-3p in bladder cancer tissues. (G) MAFG-AS1 silencing could upregulate the expression of miR-143-3p in 5367 and T24 cells. *, $\mathrm{P}<0.05$; **, $\mathrm{P}<0.01,{ }^{* * *}, \mathrm{P}<0.001$.

overexpression alleviated MAFG-AS1 depletion-mediated downregulation in the SERPINE1 expression (Figure $5 A$ ). Moreover, we observed that MAFG-AS1 depletion-impeded cells proliferation and growth were obviously attenuated by miR-143-3p inhibitors or SERPINE1 overexpression (Figure 5B,C). Wound scratch and Transwell invasion assay showed that suppressed cell migration and invasion mediated by MAFG-AS1 silencing were significantly 


\section{A Wt-SERPINE1 5, ...AAGAAACACCCTTTCATCTCA... 3, \\ miR-143-3p 3, CUCGAUGUCACGAAGUAGAGU 5, \\ Mut-SERPINE1 $5, \ldots$...AGAAACACCCTAAGUAGAGA ... 3 ,}
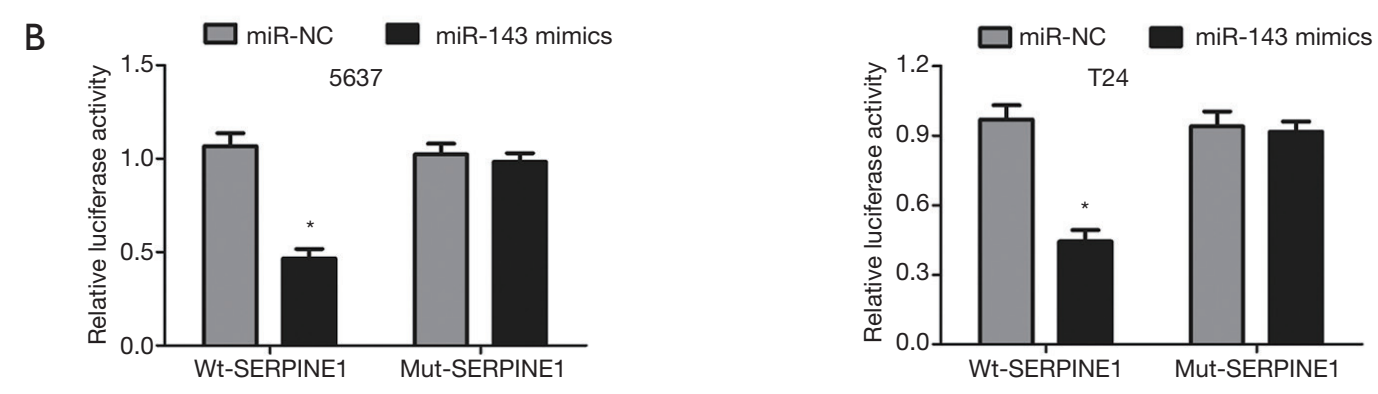

C

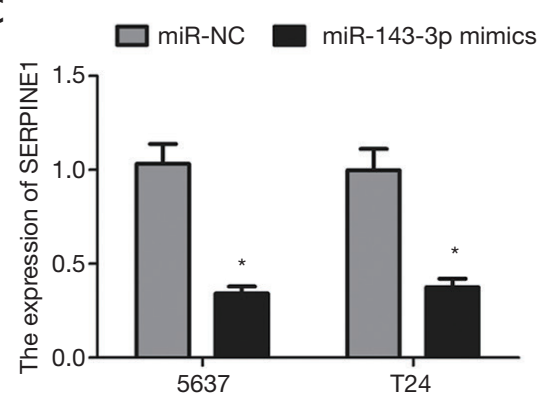

D

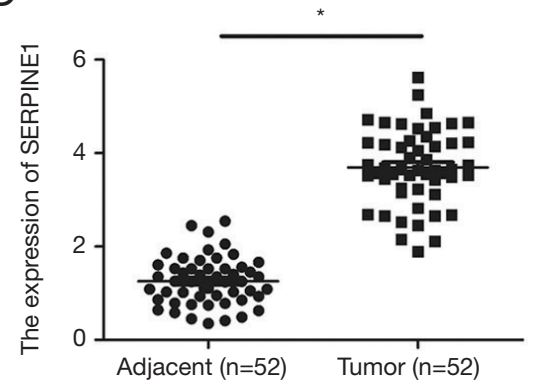

E

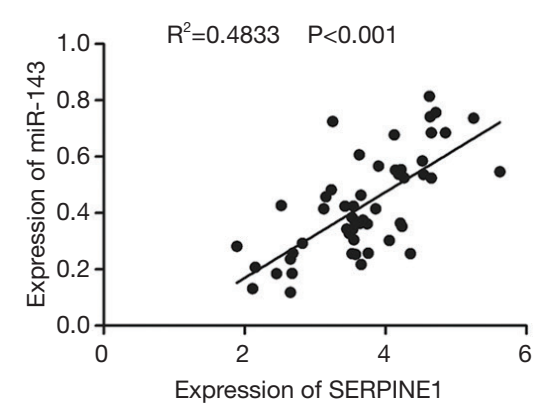

Figure 4 SERPINE1 was a target of miR-143-3p. (A) Bioinformatics predicted the possible binding site for miR-43-3p in SERPINE1. (B) Luciferase reporter assay analyzed the luciferase activity of the Wt or Mut SERPINE1 reporter in 5637 and T24 cells after transfection of miR-143-3p. (C) The expression of SERPIN1 was markedly reduced in 5367 and T24 cells transfected with miR-143-3p mimics. (D) qRTPCR detected SERPINE1 level in the 52 pairs of bladder cancer samples and matched adjacent normal tissues. (E) Correlation analysis between SERPINE1 and miR-143-3p in bladder cancer tissues. * $\mathrm{P}<0.05$.

alleviated by miR-143-3p inhibition or SERPINE1 overexpression (Figure $5 D, E$ ). Our finding indicated that MAFG-AS1 played its pro-oncogenic roles in bladder cancer via regulating miR-143-3p-SERPINE1 axis.

\section{Discussion}

Studies have shown that numerous lncRNAs are involved in tumorigenesis and have the potential to be used as biomarker in bladder cancer (6-8). For example, lncRNA OIP5-AS1 was considered as a useful biomarker for clinical progression and poor prognosis (19). LncRNA ATB promoted bladder cancer cell proliferation, migration, and invasion by regulating miR-126, and it was regarded as a potential prognostic biomarker and therapeutic target for bladder cancer (20). TUG1 facilitates bladder cancer cell growth and chemoresistance by modulating CCND2 via EZH2-associated silencing of miR-194-5p, which suggested that TUG1 can be used as a novel therapeutic target and biomarker for bladder cancer (21). It can be seen that lncRNAs have crucial role in the development of bladder cancer, and it is worthwhile to focus on the function and underlying mechanism of lncRNAs. The present study found that MAFG-AS1 was upregulated in bladder cancer samples and cell lines. MAFG-AS1 knockdown suppressed bladder cancer cell proliferation, migration and invasion. We investigated the mechanisms involved and found that MAFG-AS1 exerted its pro-oncogenic roles via competitive inhibition of miR-143-3p and elevation of SERPIN1 levels. Overall, our study revealed the role and mechanism of 
A

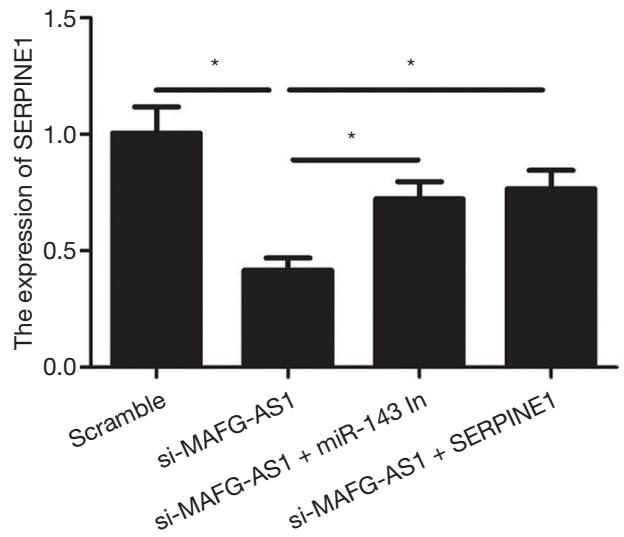

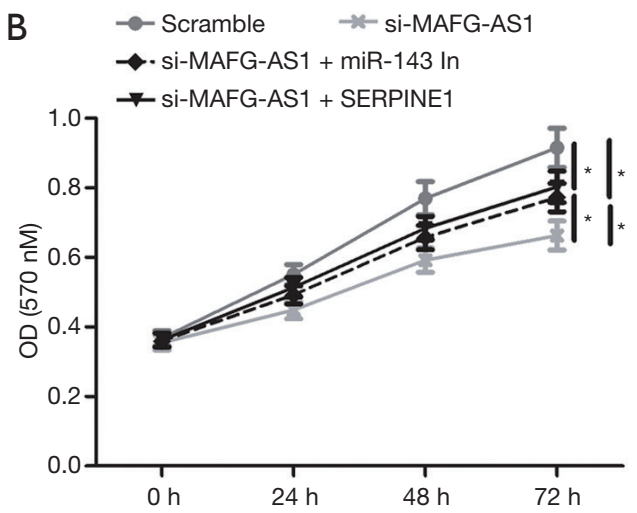

C
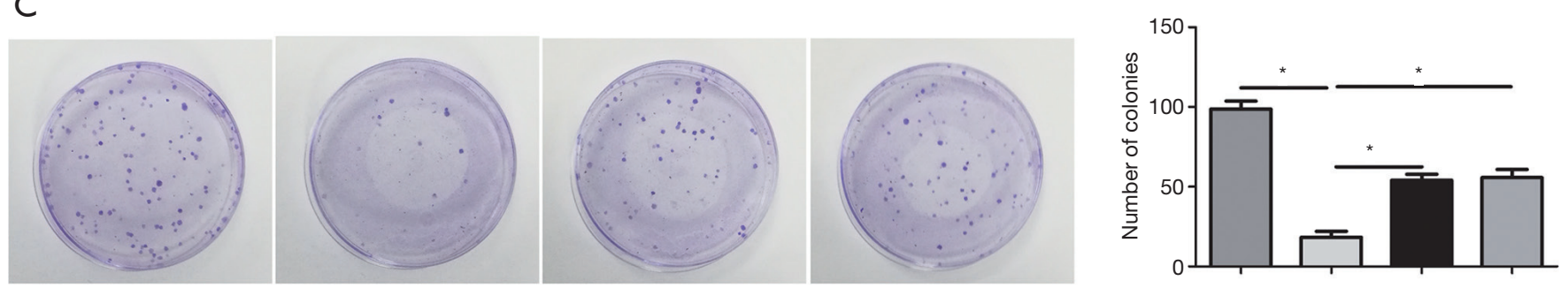

D
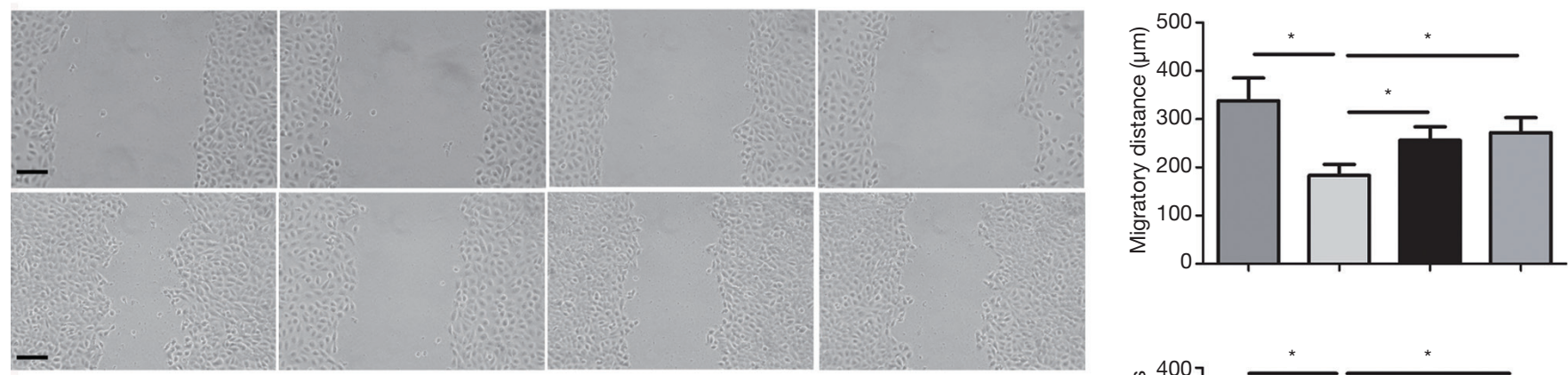

$\mathrm{E}$
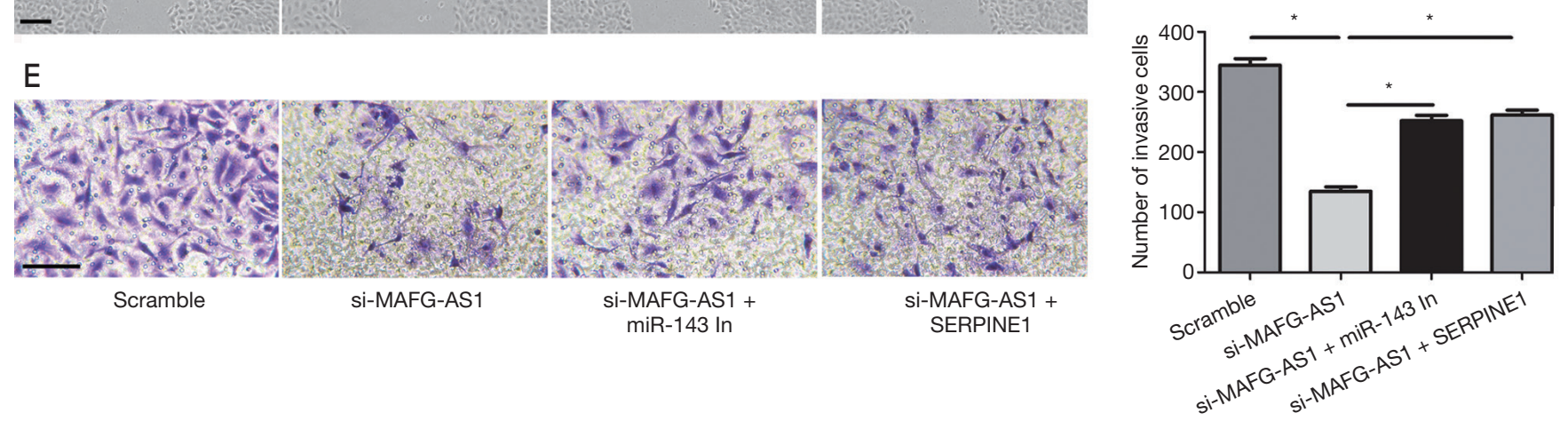

Figure 5 MAFG-AS1 facilitated bladder cells tumorigenesis by regulating miR-143-3p/SERPINE1 axis. (A) qRT-PCR showed that miR-143-3p inhibition or SERPINE1 overexpression alleviated the MAFG-AS1 silencing-induced suppressed effect on the SERPINE1 expression. MTT (B) and colony formation assay (C) showed that MAFG-AS1 depletion-impeded cells proliferation and growth were obviously attenuated by miR-143-3p inhibitors or SERPINE1 overexpression. Wound scratch (D) and Transwell invasion assay (E) showed that suppressed cell migration and invasion mediated by MAFG-AS1 silencing were significantly alleviated by miR-143-3p inhibition or SERPINE1 overexpression. Scale bars $=100 \mu \mathrm{m} .{ }^{*}, \mathrm{P}<0.05$. 
the MAFG-AS1/miR-143-3p-SERPINE1 axis in bladder cancer progression.

MAFG-AS1 was reported to be upregulated and play an oncogene role in several common carcinomas, such as colorectal cancer, breast cancer, lung cancer, hepatocellular carcinoma and gastric cancer (12-16); however, its role in bladder cancer remains unclear. We observed that MAFGAS1 was upregulated in bladder cancer tissue samples and cell lines. Further analysis revealed that MAFG-AS1 expression was positively correlated with histological grade, TNM stage and LN metastasis. Moreover, patients with high expression of MAFG-AS1 showed reduced survival. As confirmed by a series of cell function experiments in vitro, MAFG-AS1 silencing markedly suppressed bladder cancer cell malignant phenotypes. In agreement with previous reports in other carcinomas, these results confirmed that MAFG-AS1 plays an oncogene role in bladder cancer.

Many studies have reported that lncRNAs perform their roles by acting as miRNA sponges and de-suppressing their targeting mRNAs (19). Thus, the lncRNA/miRNA/ mRNA signal axis presents the most canonical model (22). For example, lncRNA HOTAI contributes to angiogenesis by modulating the miR-126/SCEL axis during burn wound healing (23). It was reported that lncRNA XIST promotes thyroid cancer cell proliferation and tumour growth by sponging miR-34a to regulate MET expression (24). Zhao et al. (25) demonstrated that IncRNA TUSC8 suppressed breast cancer progression by regulating the miR-190b-5p/MYLIP axis. In the present study, we confirmed that MAFG-AS1 performed its functions by sponging miR-143-3p to upregulate SERPINE1 levels. It was reported that miR-143-3p acts as a tumour suppressor in various carcinomas; for example, miR-143-3p impeded osteosarcoma progression by repressing stemness cell properties via targeting pro-oncogene KIA1429 (17). In breast cancer, miR-143-3p suppressed cell proliferation and invasion by targeting pyruvate carboxylase (18). In bladder cancer, miR-143-3p also was confirmed to be a tumour suppressor as it could inhibit cell growth by modulating Akt and ERK5 (26,27). Moreover, lncRNA FOXD2AS1 enhanced gemcitabine resistance via sponging miR143 in bladder cancer (22). It is known that miRNAs may have more than one target gene (28), and we found that SERPINE1 was the target gene of miR-143-3p. Moreover, the miR-143-3p/SERPIN1 axis was involved in bladder cancer progression.

SERPINE1 is a serine protease inhibitor that is mainly involved in suppression of tissue and urokinase-type plasminogen activators (29). SERPINE1 and urokinasetype plasminogen activator participated in tumour metastasis, as it proteolytically degraded the extracellular matrix (29). Numerous studies have reported that SERPINE1 dysregulation played an oncogenic role in various carcinomas; for example, SERPINE1 promoted cell proliferation, migration and invasion by modulating epithelial-mesenchymal transition in gastric cancer (29). Arroyo-Solera et al. revealed that SERPINE1 overexpression increased cell proliferation and promoted invasiveness and metastasis in head and neck squamous cell carcinoma (30). In osteosarcoma, SERPINE1 was shown to facilitate cell invasion and metastasis by upregulating MMP-13 (31). In bladder cancer, overexpression of SERPINE1 was closely correlated with bladder urothelial carcinoma aggressiveness and/or poor survival, but also contributed to cell invasiveness (32). In the present study, we revealed that SERPINE1 was overexpressed in bladder cancer tissues and acted as a target gene of miR-143-3p. We also showed that SERPINE1 is involved in the modulation of MAFG-AS1 in bladder cancer proliferation, migration and invasion.

Taken together, our results indicate that MAFG-AS1 is overexpressed in bladder cancer. MAFG-AS1 silencing inhibited bladder cancer cell proliferation, migration and invasion by modulating the miR-143-SERPINE1 axis. Our findings provide a novel insight into tumorigenesis and reveal a promising therapeutic target for bladder cancer.

\section{Acknowledgments}

Funding: This work was supported by the National Youth Science Foundation of China (81802561).

\section{Footnote}

Reporting Checklist: The authors have completed the MDAR checklist. Available at http://dx.doi.org/10.21037/ tcr-20-1971

Data Sharing Statement: Available at http://dx.doi. org/10.21037/tcr-20-1971

Conflicts of Interest: All authors have completed the ICMJE uniform disclosure form (available at http://dx.doi. org/10.21037/tcr-20-1971). The authors have no conflicts of interest to declare.

Ethical Statement: The authors are accountable for all 
aspects of the work in ensuring that questions related to the accuracy or integrity of any part of the work are appropriately investigated and resolved. The study was conducted in accordance with the Declaration of Helsinki (as revised in 2013). The study was approved by the institutional ethics committee of Xiangya Hospital, Central South University (approval No. 2019032542), and written informed consents were obtained from all patients.

Open Access Statement: This is an Open Access article distributed in accordance with the Creative Commons Attribution-NonCommercial-NoDerivs 4.0 International License (CC BY-NC-ND 4.0), which permits the noncommercial replication and distribution of the article with the strict proviso that no changes or edits are made and the original work is properly cited (including links to both the formal publication through the relevant DOI and the license). See: https://creativecommons.org/licenses/by-nc-nd/4.0/.

\section{References}

1. Fujii Y. Prediction models for progression of nonmuscle-invasive bladder cancer: A review. Int J Urol 2018;25:212-8.

2. Qiu F, Zhang MR, Zhou Z, et al. lncRNA MIR503HG functioned as a tumor suppressor and inhibited cell proliferation, metastasis and epithelial-mesenchymal transition in bladder cancer. J Cell Biochem 2019;120:10821-9.

3. Oughton JB, Poad H, Twiddy M, et al. Radical cystectomy (bladder removal) against intravesical BCG immunotherapy for high-risk non-muscle invasive bladder cancer (BRAVO): a protocol for a randomised controlled feasibility study. BMJ Open 2017;7:e017913.

4. Yu C, Longfei L, Long W, et al. LncRNA PVT1 regulates VEGFC through inhibiting miR-128 in bladder cancer cells. J Cell Physiol 2019;234:1346-53.

5. Mercer TR, Dinger ME, Mattick JS. Long noncoding RNAs: insights into functions. Nat Rev Genet 2009;10:155-9.

6. He A, He S, Peng D, et al. Prognostic value of long noncoding RNA signatures in bladder cancer. Aging (Albany NY) 2019;11:6237-51.

7. Jiang $\mathrm{L}, \mathrm{Li}$ Z, Wang R. Long noncoding RNAs in lung cancer: Regulation patterns, biologic function and diagnosis implications (Review). Int J Oncol 2019;55:585-96.

8. Luo L, Wang M, Li X, et al. Long non-coding RNA LOC285194 in cancer. Clin Chim Acta 2020;502:1-8.
9. Chen $\mathrm{C}, \mathrm{He} \mathrm{W}$, Huang J, et al. LNMAT1 promotes lymphatic metastasis of bladder cancer via CCL2 dependent macrophage recruitment. Nat Commun 2018;9:3826.

10. Chen JB, Zhu YW, Guo X, et al. Microarray expression profiles analysis revealed IncRNA OXCT1-AS1 promoted bladder cancer cell aggressiveness via miR-455-5p/JAK1 signaling. J Cell Physiol 2019;234:13592-601.

11. Dai R, Zhou Y, Chen Z, et al. Lnc-MUC20-9 binds to ROCK1 and functions as a tumor suppressor in bladder cancer. J Cell Biochem 2020;121:4214-25.

12. Cui S, Yang X, Zhang L, et al. LncRNA MAFG-AS1 promotes the progression of colorectal cancer by sponging miR-147b and activation of NDUFA4. Biochem Biophys Res Commun 2018;506:251-8.

13. Li C, Wu R, Xing Y. MAFG-AS1 is a novel clinical biomarker for clinical progression and unfavorable prognosis in gastric cancer. Cell Cycle 2020;19:601-9.

14. Li H, Zhang GY, Pan CH, et al. LncRNA MAFG-AS1 promotes the aggressiveness of breast carcinoma through regulating miR-339-5p/MMP15. Eur Rev Med Pharmacol Sci 2019;23:2838-46.

15. Ouyang H, Zhang L, Xie Z, et al. Long noncoding RNA MAFG-AS1 promotes proliferation, migration and invasion of hepatocellular carcinoma cells through downregulation of miR-6852. Exp Ther Med 2019;18:2547-53.

16. Sui $Y$, Lin G, Zheng Y, et al. LncRNA MAFG-AS1 boosts the proliferation of lung adenocarcinoma cells via regulating miR-744-5p/MAFG axis. Eur J Pharmacol 2019;859:172465.

17. Han Q, Yang J, Yang H, et al. KIAA1429 promotes osteosarcoma progression by promoting stem cell properties and is regulated by miR-143-3p. Cell Cycle 2020;19:1172-85.

18. Pinweha P, Phillips CA, Gregory PA, et al. MicroRNA143-3p targets pyruvate carboxylase expression and controls proliferation and migration of MDA-MB231cells. Arch Biochem Biophys 2019;677:108169.

19. Yang J, Jiang B, Hai J, et al. Long noncoding RNA opainteracting protein 5 antisense transcript 1 promotes proliferation and invasion through elevating integrin alpha6 expression by sponging miR-143-3p in cervical cancer. J Cell Biochem 2019;120:907-16.

20. Zhai $X, X u$ W. Long Noncoding RNA ATB Promotes Proliferation, Migration, and Invasion in Bladder Cancer by Suppressing MicroRNA-126. Oncol Res 2018;26:1063-72. 21. Yu G, Zhou H, Yao W, et al. lncRNA TUG1 Promotes 
Cisplatin Resistance by Regulating CCND2 via Epigenetically Silencing miR-194-5p in Bladder Cancer. Mol Ther Nucleic Acids 2019;16:257-71.

22. An Q, Zhou L, Xu N. Long noncoding RNA FOXD2AS1 accelerates the gemcitabine-resistance of bladder cancer by sponging miR-143. Biomed Pharmacother 2018;103:415-20.

23. Jiang B, Tang $\mathrm{Y}$, Wang $\mathrm{H}$, et al. Down-regulation of long non-coding RNA HOTAIR promotes angiogenesis via regulating miR-126/SCEL pathways in burn wound healing. Cell Death Dis 2020;11:61.

24. Liu H, Deng H, Zhao Y, et al. LncRNA XIST/miR-34a axis modulates the cell proliferation and tumor growth of thyroid cancer through MET-PI3K-AKT signaling. J Exp Clin Cancer Res 2018;37:279.

25. Zhao L, Zhou Y, Zhao Y, et al. Long non-coding RNA TUSC8 inhibits breast cancer growth and metastasis via miR-190b-5p/MYLIP axis. Aging (Albany NY) 2020;12:2974-91.

26. Noguchi S, Mori T, Hoshino Y, et al. MicroRNA-143 functions as a tumor suppressor in human bladder cancer T24 cells. Cancer Lett 2011;307:211-20.

27. Noguchi S, Yasui Y, Iwasaki J, et al. Replacement treatment with microRNA-143 and -145 induces synergistic inhibition of the growth of human bladder cancer cells by regulating PI3K/Akt and MAPK signaling pathways. Cancer Lett 2013;328:353-61.

28. An Y, Furber KL, Ji S. Pseudogenes regulate parental gene expression via ceRNA network. J Cell Mol Med 2017;21:185-92.

29. Yang JD, Ma L, Zhu Z. SERPINE1 as a cancer-promoting gene in gastric adenocarcinoma: facilitates tumour cell proliferation, migration, and invasion by regulating EMT. J Chemother 2019;31:408-18.

30. Arroyo-Solera I, Pavon MA, Leon X, et al. Effect of serpinE1 overexpression on the primary tumor and lymph node, and lung metastases in head and neck squamous cell carcinoma. Head Neck 2019;41:429-39.

31. Hirahata M, Osaki M, Kanda Y, et al. PAI-1, a target gene of miR-143, regulates invasion and metastasis by upregulating MMP-13 expression of human osteosarcoma. Cancer Med 2016;5:892-902.

32. Li X, Dong P, Wei W, et al. Overexpression of CEP72 Promotes Bladder Urothelial Carcinoma Cell Aggressiveness via Epigenetic CREB-Mediated Induction of SERPINE1. Am J Pathol 2019;189:1284-97.
Cite this article as: Sun X, Cai Y, Hu X, Mo M, Zhao C, He W, Li Y. Long noncoding RNA MAFG-AS1 facilitates bladder cancer tumorigenesis via regulation of miR-143-3p/SERPINE1 axis. Transl Cancer Res 2020;9(11):7214-7226. doi: 10.21037/tcr20-1971 